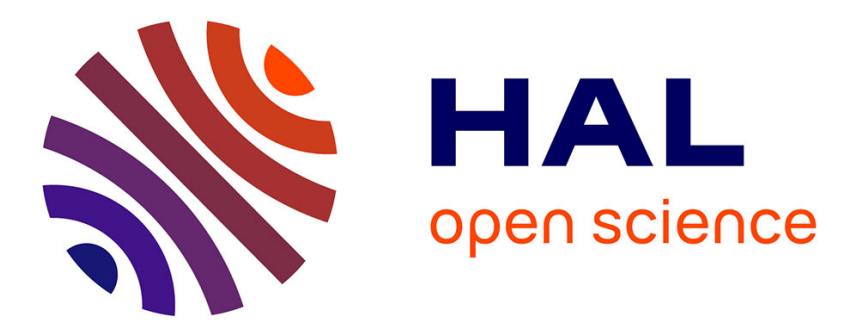

\title{
Bacteremia Due to Kosakonia cowanii in a Preterm Neonate
}

\author{
Claire Duployez, Marie-Eve Edun-Renard, Eric Kipnis, Rodrigue Dessein, \\ Rémi Le Guern
}

\section{- To cite this version:}

Claire Duployez, Marie-Eve Edun-Renard, Eric Kipnis, Rodrigue Dessein, Rémi Le Guern. Bacteremia Due to Kosakonia cowanii in a Preterm Neonate. Journal of Pediatric Infectious Diseases, 2021, 16 (04), pp.183-186. 10.1055/s-0040-1721448 . hal-03309806

\section{HAL Id: hal-03309806 https://hal.science/hal-03309806}

Submitted on 30 Jul 2021

HAL is a multi-disciplinary open access archive for the deposit and dissemination of scientific research documents, whether they are published or not. The documents may come from teaching and research institutions in France or abroad, or from public or private research centers.
L'archive ouverte pluridisciplinaire HAL, est destinée au dépôt et à la diffusion de documents scientifiques de niveau recherche, publiés ou non, émanant des établissements d'enseignement et de recherche français ou étrangers, des laboratoires publics ou privés. 
1 TITLE: Bacteremia due to Kosakonia cowanii in a preterm neonate.

2

3 AUTHORS: Claire Duployez ${ }^{1,2}$, Marie-Eve Edun-Renard ${ }^{3}$, Eric Kipnis ${ }^{2,4}$, Rodrigue 4 Dessein $^{1,2}$, Rémi Le Guern ${ }^{1,2}$

5

6 AUTHORS AFFILIATIONS:

$7 \quad{ }^{1}$ Laboratoire de Bactériologie, CHU Lille, F-59000 Lille, France.

$8 \quad{ }^{2}$ Univ. Lille, CNRS, Inserm, CHU Lille, Institut Pasteur de Lille, U1019 - UMR 9017 - CIIL

9 - Center for Infection and Immunity of Lille, F-59000 Lille, France.

${ }^{3}$ Unité des Soins Intensifs, Service de Néonatalogie, CHU Lille, F-59000 Lille, France.

${ }^{4}$ Pôle d'Anesthésie-Réanimation, CHU Lille, F-59000 Lille, France.

\section{CORRESPONDING AUTHOR: Dr Claire DUPLOYEZ}

claire.duployez@chru-lille.fr

Laboratoire de Bactériologie-Institut de Microbiologie

Centre de Biologie Pathologie

F-59037-Lille Cedex, France

Telephone number: $+33-320.445 .480$

Fax number: +33-320.444.895 


\section{ABSTRACT}

Objective: Low-weight birth infants admitted to neonatal intensive care units are at high risk of hospital-acquired infections by opportunistic pathogens. The gut microbiota of preterm neonates lacks commensal bacteria providing a barrier against pathogens. We report a case of bacteremia due to Kosakonia cowanii in a preterm neonate.

Case report: Caesarian-section delivered a female baby of $680 \mathrm{~g}$ at 28 weeks of gestation due to intrauterine growth retardation and fetal rhythm abnormalities. On day 27, two blood cultures grew Gram-negative bacilli in a context of functional ileus. No reliable identification could be obtained using MALDI-TOF mass spectrometry, biochemical reactions with the VITEK 2 GN ID card, or 16S rDNA sequencing. Kosakonia cowanii was finally identified by $\operatorname{gyr} B$ sequencing. The source of infection may have been either the central venous catheter or translocation from the gut microbiota. Evolution was favorable after 14 days of cefepime (combined with amikacin for five days) and central venous catheter removal.

Conclusion: Kosakonia cowanii is a member of the Enterobacteriaceae family that was recently reclassified from the Enterobacter genus. Human infections due to $K$. cowanii are scarce and have mainly been associated with traumatic inoculation from plants or transient gut colonization. $K$. cowanii may be an underestimated opportunistic pathogen in susceptible populations such as preterm neonates.

KEY-WORDS: Bacteriological Techniques; Kosakonia cowanii; Prematurity 


\section{Introduction}

Healthcare-associated infections frequently occur in neonatal intensive care units, with a peak incidence reported between the $10^{\text {th }}$ and $22^{\text {nd }}$ day of life ${ }^{1}$. Incidence of late-onset sepsis is inversely correlated to birth weight: $36.3 \%$ of neonates with a gestational age under 28 weeks had at least one episode of sepsis compared to $29.6 \%$ for moderately preterm, $17.5 \%$ for late preterm, and $16.5 \%$ for full term infants ${ }^{1}$. Risk factors include the immature innate immune system of preterm neonates, prolonged hospitalization, use of invasive medical procedures, and failure of early enteral feeding with breast milk ${ }^{1,2}$. Moreover, the gut microbiota of preterm neonates lacks commensal bacteria providing resistance against pathogens ${ }^{3}$. Thus, the risk of infection due to translocation from the intestinal tract may increase. Indeed, Stewart et al. found that the the causative agent of late-onset sepsis in preterm neonates was within the dominant genus of the gut microbiome ${ }^{4}$. Enterobacter species are the most abundant bacteria present in preterm neonates' gut, especially after antimicrobial therapy ${ }^{5}$. Kosakonia cowanii is a member of the Enterobacteriaceae family that was recently reclassified from the Enterobacter genus ${ }^{6} . K$. cowanii is frequently encountered as a plant pathogen but rarely described in human infections. We present a case of bacteremia due to $K$. cowanii in a preterm neonate.

\section{Case report}

Caesarian-section delivered a female baby of $680 \mathrm{~g}$ at 28 weeks of gestation due to intrauterine growth retardation and fetal rhythm abnormalities. A central venous catheter was inserted, and enteral nutrition with pasteurized breast milk was initiated on day 1 of life. On day 10, the neonate presented bacterial sepsis due to Staphylococcus epidermidis : he had fever at $38^{\circ} \mathrm{C}$, frequent apneas with bradycardia and desaturation. Continuous Positive Airway Pressure 
(CPAP) and transfusion of one unit of packed red blood cells were required. The evolution was favorable after ten days of antimicrobial therapy with vancomycin.

On day 27 , the baby presented abdominal distension with residual feeds attributed to a functional ileus, since she had no rectal bleeding nor vomiting. Hemodynamic and respiratory functions deteriorated again, requiring endotracheal intubation from day 28 to day 31 , transfusion of five units of packed red blood cells and daily platelet transfusion from day 27 to day 34. Enteral feeding was temporarily stopped.

Blood cultures from day 27 yielded Gram-negative bacilli after 16 hours of incubation. No reliable identification was assessed by MALDI-TOF spectrometry mass (Bruker Daltonics, Wissembourg, France); the best score was 1.54 for $K$. cowanii. Biochemical reactions using VITEK 2 GN ID card (BioMérieux, Marcy l'Etoile, France) identified the strain as Pantoea spp., with a probability of $97 \%$. However, the strain fermented sorbitol and did not use malonate; those two characteristics distinguish $K$. cowanii from Pantoea agglomerans. Sequencing of $16 \mathrm{~S}$ rDNA was performed using universal primers 91E (TCAAAKGAATTGACGGGGGC-3) and 13BS (CCCGGGAACGTATTCAC) on the 3500 Dx Genetic Analyzer (Life Technologies, Cergy-Pontoise, France) ${ }^{7}$. Definitive identification could not be obtained from the 16S partial sequence, because $99 \%$ identity out of 431 base pairs was found for K. cowanii, Salmonella enterica, and Atlantibacter hermannii. Thus, gyrB sequencing was performed using the primers UP1 (GAAGTCATCATGACCGTTCTGCAYGCNGGNGGNAARTTYGA) and 181r (CAGGAAACAGCTATGACCARRTGNGTNCCNCC) for amplification, followed by UP1seq (GAAGTCATCATGACCGTTCTGCA) and 181r-seq (CAGGAAACAGCTATGACC) for sequencing ${ }^{8} \cdot g y r B$ sequencing identified the isolate as $K$. cowanii, showing $99 \%$ identity out of 521 base pairs with $K$. cowanii type strain JCM 10956 (Genebank accession number 
JX425012.1). A phylogenetic tree based on gyrB sequences (Figure 1) was constructed in MEGA $X^{9}$, using the minimum evolution method.

In vitro antimicrobial susceptibility was determined by Mueller-Hinton agar disc diffusion using the critical zone diameters recommended by the CASFM -EUCAST (version 2020) and using an automated method (VITEK 2, BioMérieux, Marcy 1'Etoile, France). Interestingly, this strain did not produce an AmpC $\beta$-lactamase as expected from a former Enterobacter species, but a penicillinase. The strain was resistant to amoxicillin (MIC $\geq 32 \mathrm{mg} / \mathrm{L}$ ), ticarcillin (MIC $\geq 128 \mathrm{mg} / \mathrm{L})$, and piperacillin $(\mathrm{MIC}=64 \mathrm{mg} / \mathrm{L})$; all the other beta-lactams were active, including amoxicillin-clavulanate (MIC $\leq 2 \mathrm{mg} / \mathrm{L}$ ) and cephalosporins (MIC $\leq 1$ $\mathrm{mg} / \mathrm{L}$ for cefotaxime, ceftriaxone, and cefepime).

After 14 days of cefepime, initiated on day 27 (combined with amikacin for five days) and central venous catheter removal, evolution was favorable. Antimicrobial therapy and laboratory values are represented as a timeline in Figure 2. Blood cultures remained sterile after catheter change; $K$. cowanii also grew from the central venous catheter $\left(10^{3} \mathrm{CFU} / \mathrm{mL}\right)$, indicating it could be the infection source. The neonate was discharged from the hospital after three months of life, weighing $2615 \mathrm{~g}$ for $48 \mathrm{cms}$.

\section{Discussion}

Until 2013, the Kosakonia genus was part of the genus Enterobacter. Many species from the Kosakonia genus (K. radicincitans, $K$. sacchari, K. oryzae, $K$. cowanii, $K$. arachidis) have been isolated from plants, and only a few human infections have been described ${ }^{10}$. K. cowanii was first described from a patient sample but is mostly isolated from environmental samples, including soil and trees. $K$. cowanii may promote plant growth and be identified as an endophyte in trees with symptoms of blight ${ }^{6}$. Human infections due to K. cowanii are scarce. They have been associated with traumatic inoculation from plants. In a 
case, rhabdomyolysis due to bacteremia was observed after rose thorn prick ${ }^{11}$. More recently, Berinson et al. reported a case of acute cholecystitis in an immunocompromised patient without penetrating trauma ${ }^{12}$, suggesting the origin of the infection may be food contamination. The authors suggested that transient colonization of the gut by $K$. cowanii may have caused acute cholecystitis in an immunocompromised patient.

Low-weight birth infants admitted in neonatal intensive care units are at high risk of hospital-acquired infections by opportunistic pathogens ${ }^{2}$. No case of $K$. cowanii opportunistic infections in low-weight birth infants had been reported in the literature yet. Our case report highlights that $K$. cowanii may be a relevant cause of infections in the population of preterm neonates. Preterm neonates are vulnerable to infections caused by opportunistic foodborne pathogens since their local natural barrier is immature, and secretory immunoglobulins $\mathrm{A}$ are lacking during the first days of life. $K$. cowanii was previously isolated from powdered infant milk formula ${ }^{13}$. However, food contamination may not be the origin of infection in our case since the neonate was fed with pasteurized breast milk. In our patient, the source of infection may have been bacterial translocation from the gut microbiota. Indeed, previous studies showed that the pathogen identified in blood culture for late-onset sepsis in preterm infants was usually abundant in the gut microbiome ${ }^{4}$. Additional risk factors of bacterial sepsis in this population include a central venous catheter, parenteral nutrition, and steroids ${ }^{14}$. Another hypothesis for the infection source may have been the central venous catheter, as suggested by the positive bacteriological culture of the catheter.

Other environmental species, mostly former members of the Enterobacter group, were described as pathogens for neonates. The typical example is Cronobacter sakazakii, which has been isolated from environmental sources, milk, and infant formula, causing severe infections, especially bacteremia in preterm neonates ${ }^{6,15}$. Powdered infant formula has been reported as the most commonly identified transmission vehicle of this bacterium ${ }^{15}$. 
Berinson et al. suggested that $K$. cowanii might be an underestimated pathogen in 143 children $^{12}$. Indeed, in a retrospective study about pediatric infections due to 144 Pantoea agglomerans, seven patients out of $53(13 \%)$ had a history of penetrating trauma 145 with a stick, plant thorn, or glass shard ${ }^{16}$. As shown in our case report, $K$. cowanii may be 146 falsely identified as P. agglomerans using biochemical reactions. It may be one of the reasons 147 for the few human infections described in the literature. The development of new 148 bacteriological techniques such as MALDI-TOF mass spectrometry and molecular biology 149 may improve our ability to identify this bacterium. $K$. cowanii is probably an underestimated 150 opportunistic pathogen in susceptible populations such as preterm neonates.

Acknowledgments: We thank Laure Marceau for technical help with DNA sequencing. 


\section{REFERENCES}

1. Dong Y, Speer CP. Late-onset neonatal sepsis: recent developments. Arch Dis Child Fetal Neonatal Ed. 2015 May;100(3):F257-63.

2. Sharma AA, Jen R, Butler A, et al. The developing human preterm neonatal immune system: a case for more research in this area. Clin Immunol 2012;145(1):61-8.

3. Drell T, Lutsar I, Stsepetova J, et al. The development of gut microbiota in critically ill extremely low birth weight infants assessed with $16 \mathrm{~S}$ rRNA gene based sequencing. Gut Microbes 2014;5(3):304-12.

4. Stewart CJ, Embleton ND, Marrs ECL, et al. Longitudinal development of the gut microbiome and metabolome in preterm neonates with late onset sepsis and healthy controls. Microbiome. 2017 Jul 12;5(1):75.

5. Greenwood C, Morrow AL, Lagomarcino AJ, et al. Early empiric antibiotic use in preterm infants is associated with lower bacterial diversity and higher relative abundance of Enterobacter. J Pediatr 2014;165(1):23-9.

6. Brady C, Cleenwerck I, Venter S, et al. Taxonomic evaluation of the genus Enterobacter based on multilocus sequence analysis (MLSA): proposal to reclassify E. nimipressuralis and E. amnigenus into Lelliottia gen. nov. as Lelliottia nimipressuralis comb. nov. and Lelliottia amnigena comb. nov., respectively, E. gergoviae and E. pyrinus into Pluralibacter gen. nov. as Pluralibacter gergoviae comb. nov. and Pluralibacter pyrinus comb. nov., respectively, E. cowanii, E. radicincitans, E. oryzae and E. arachidis into Kosakonia gen. nov. as Kosakonia cowanii comb. nov., Kosakonia radicincitans comb. nov., Kosakonia oryzae comb. nov. and Kosakonia arachidis comb. nov., respectively, and E. turicensis, E. helveticus and E. pulveris into Cronobacter as Cronobacter zurichensis nom. nov., Cronobacter helveticus comb. nov. 
and Cronobacter pulveris comb. nov., respectively, and emended description of the genera Enterobacter and Cronobacter. Syst Appl Microbiol 2013;36(5):309-19.

7. Le Guern R, Loïez C, Armand S, et al. Infective endocarditis: does a new 16S rDNA set of primers improve the microbiological diagnosis? Infect Dis (Lond). 2015;47(12):896-901.

8. Delmas J, Breysse F, Devulder G, et al. Rapid identification of Enterobacteriaceae by sequencing DNA gyrase subunit B encoding gene. Diagn Microbiol Infect Dis. 2006 Aug;55(4):263-8.

9. Kumar S., Stecher G., Li M., et al. MEGA X: Molecular Evolutionary Genetics Analysis across computing platforms. Molecular Biology and Evolution 2018;35:1547-1549.

10. Mertschnigg T, Patz S, Becker M, et al. First Report of Kosakonia radicincitans Bacteraemia from Europe (Austria) - Identification and Whole-Genome Sequencing of Strain DSM 107547. Sci Rep. 2020 Feb 6;10(1):1948.

11. Washio K, Yamamoto G, Ikemachi M, et al. Rhabdomyolysis due to bacteremia from Enterobacter cowanii caused by a rose thorn prick. J Dermatol 2018;45(11):e313-e4.

12. Berinson B, Bellon E, Christner M, et al. Identification of Kosakonia cowanii as a rare cause of acute cholecystitis: case report and review of the literature. BMC Infect Dis 2020;20(1):366.

13. Mardaneh J, Soltan-Dallal MM. Isolation and Identification of E. cowanii from Powdered Infant Formula in NICU and Determination of Antimicrobial Susceptibility of Isolates. Iran J Pediatr 2014;24(3):261-6.

14. Manzoni P, De Luca D, Stronati M, et al. Prevention of nosocomial infections in neonatal intensive care units. Am J Perinatol 2013;30(2):81-8. 
199 15. Strysko J, Cope JR, Martin H, T et al. Food Safety and Invasive Cronobacter Infections 200 during Early Infancy, 1961-2018. Emerg Infect Dis 2020;26(5).

201 16. Cruz AT, Cazacu AC, Allen CH. Pantoea agglomerans, a plant pathogen causing human 202 disease. J Clin Microbiol 2007;45(6):1989-92. 
204 Figure 1. Phylogenetic tree based on gyrB sequences.

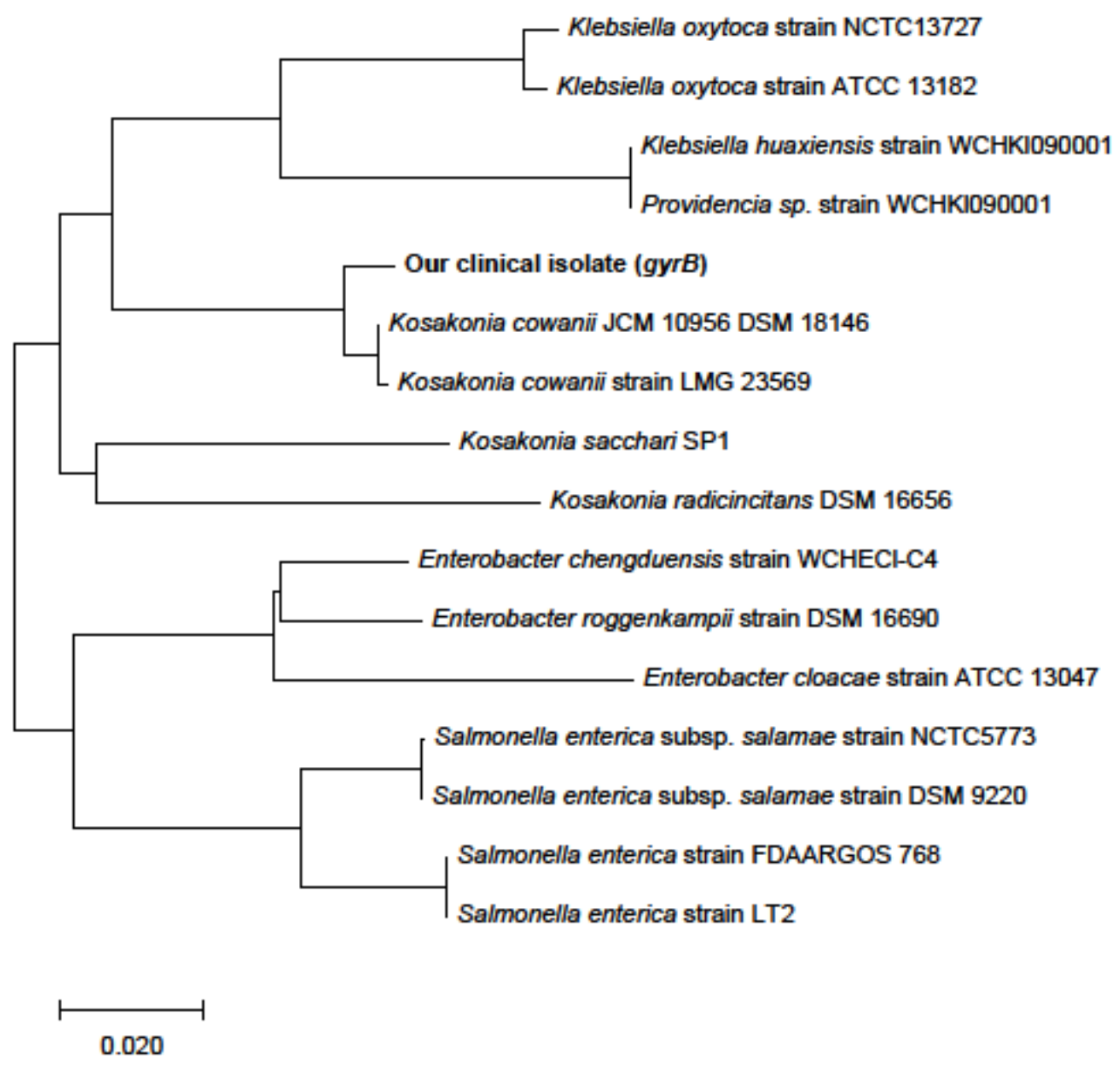


Figure 2. Timeline visualization of laboratory data and antimicrobial therapy.
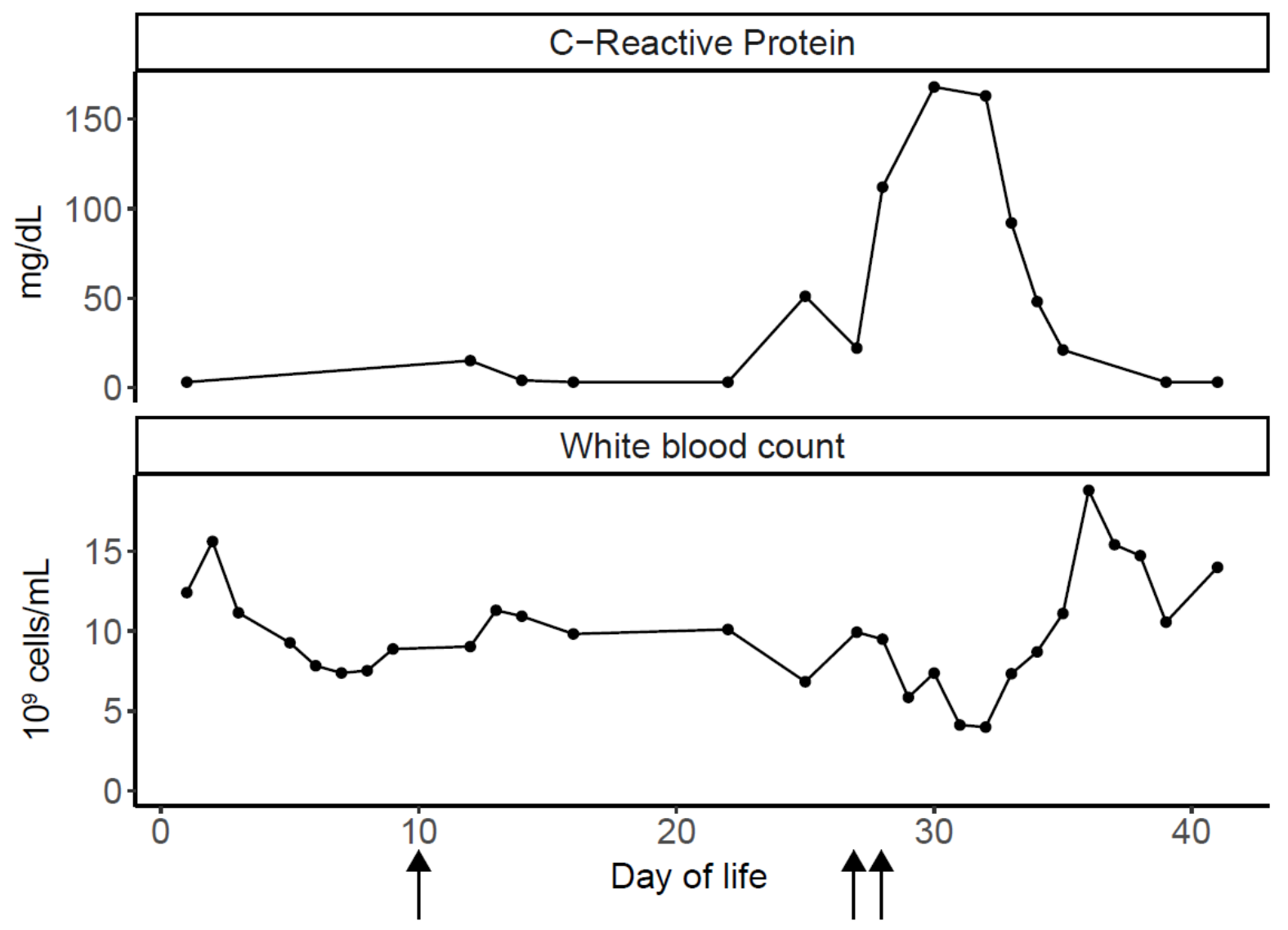

Blood cultures S. epidermidis

K. cowanii

Amikacin

Antimicrobial therapy
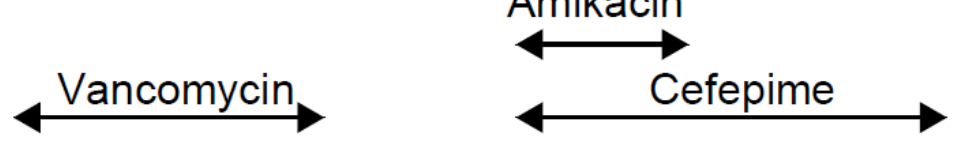